\section{Hapten-Modell contra p-i-Konzept}

\author{
Ende letzten Jahres publizierte Prof. Hans F. Merk im Themenheft \\ „Arzneimittelreaktionen“ des Allergo Journal umfassend über \\ „Allergische Arzneimittelreaktionen der Haut: Epidemiologie, Klinik \\ und Pathogenese“ (Allergo J 2006; 15: 476-91). Aus der Schweiz \\ kommen Anmerkungen zur Darstellung des pathogenetischen \\ p-i-Konzeptes.
}

$\mathrm{H}$ err Prof. Merk nimmt in seiner sehr schönen Übersichtsarbeit Stellung zum so genannten p-i-Konzept, welches unsere Arbeitsgruppe als neuen Mechanismus einer Medikamentenreaktion entwickelt hat. Das Kürzel p-i steht für „pharmacological interaction with immune receptors" und meint nichts anderes, als dass Medikamente auch direkt mit Zelloberflächenrezeptoren wie beispielsweise mit T-Zell-Rezeptoren interagieren können. Dies kann unter Umständen die TZellen stimulieren, welche dann Zytokine sezernieren und proliferieren. Dieses Konzept ergänzt die Hapten-/Prohapten-Hypothese. Prof Merk wertet das $\mathrm{p}-\mathrm{i}$ Konzept in seiner Arbeit nur als eine mögliche Erklärung für den manchmal positiven Lymphozyten-Transformationstest ohne weitere wesentliche klinische Relevanz. Dies unterschätzt meiner Meinung nach die Bedeutung des p-i Konzeptes, weshalb ich auf einige Punkte eingehen möchte:

1. Prof. Merk hat zwar selbst Daten generiert, die er mit dem p-i-Konzept interpretiert [9], dennoch bezweifelt er die Möglichkeit, mit einem Medikament eine Immunantwort zu induzieren. Das p-i-Konzept postuliert aber gar keine eigenständige Immunantwort, sondern vielmehr eine Reaktivierung Peptid-spezifischer T-Zellen [6]. Einige dieser T-Zellen können auch mit dem Medikament reagieren, und zwar mit Aktivierung. Prof. Merk behauptet, dass nicht gezeigt wurde, dass Medikamenten-spezifische T-Zell-Klone mit Peptiden reagieren, jedoch konnte von Greyerz bereits 2001 zeigen [5], dass ein relativ hoher Prozentsatz Medikamenten-spezifischer TZell-Klone auch alloreaktiv ist - ohne
Medikament - und alloreaktive T-Zellen sind Peptid-spezifisch.

2. Prof. Merk schreibt weiter, dass die meisten Medikamenten-reaktiven TLymphozyten mit Metaboliten reagieren. Dies ist falsch, eine Reaktivität mit Metaboliten ist vielmehr die Aus-

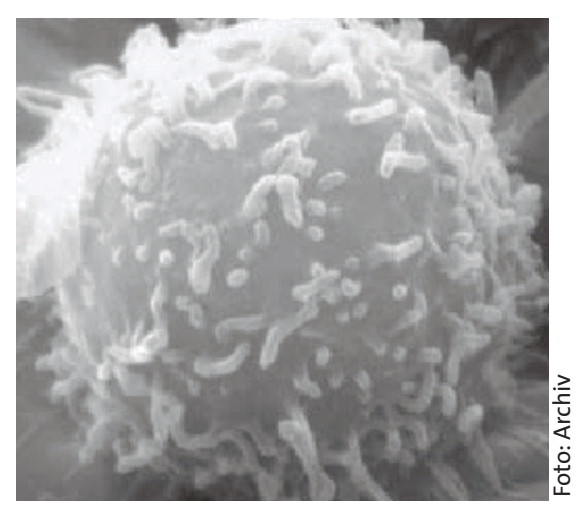

Lassen sich T-Zellen direkt mit Medikamenten stimulieren?

nahme: Nur 14 von 222 T-Zell-Klonen eines gegen Sulfamethoxazol allergischen Patienten reagierten mit dem Metaboliten SulfamethoxazolNO, dagegen 207 mit Sulfamethoxazol alleine [1, 7]. Die meisten T-ZellKlone von Medikamentenallergikern reagieren ausschließlich mit der nicht

3. Im Tiermodell der Nevirapin-Sensibilisierung reagieren die $\mathrm{T}$-Zellen nicht nur mit dem Metaboliten, sondern auch mit der unmetabolisierten Substanz, genauso wie beim Menschen [1]. Eine Blockade der Metabolisierung steigert sogar eher die klinische Manifestation [8].

4. Unter gewissen Umständen ist es sogar möglich, in vitro eine Induktion von metabolisierten Substanz. proliferierenden T-Zellen gegen das unveränderte, nicht als Hapten fungierende Medikament zu induzieren - wie wir es in vivo postulieren. Eine massive Vorstimulation der reaktiven T-Zellen ist allerdings nötig [2].

Das p-i-Konzept erklärt somit logisch und konsistent Vorgänge wie z. B. Medikamentenallergien bei Erstexposition, Hauttests auf Medikamente, die keine Haptene sind, die gleichzeitige Stimulation von $\mathrm{CD}^{+}{ }^{+}$- und $\mathrm{CD}^{+}{ }^{+}$-Medikamenten-spezifischen T-Zellen etc. (zusammengefasst in [4]). Die Tatsache, dass das $\mathrm{p}-\mathrm{i}$-Konzept in Tierexperimenten noch nicht bestätigt wurde, ist keine Widerlegung. In den gleichen Tieren wurde nach Verabreichung von Sulfamethoxazol mit Adjuvans auch kaum eine Sulfamethoxazol-NO-gerichtete Immunantwort entdeckt, sodass auch das Pro-Hapten-Konzept bei Sulfamethoxazol nicht bestätigt wurde [3]. Insgesamt sind die humanen und experimentellen Daten zum p-i Konzept bedeutend solider als die zum Prohapten-Konzept, welches ich allerdings durchaus als relevant einstufe, v. a. bei Kontaktdermatitis und bei isolierten medikamentenallergischen Hepatitiden.

Sicherlich ist es schwer einen Paradigmenwechsel vorzunehmen - aber in der Medikamentenallergie ist dieser überfällig: Medikamentennebenwirkungen werden oft den Typ-B-Reaktionen zugeordnet, wobei B gern als „bizarr“ interpretiert wurde. Allerdings sind nicht die Reaktionen bizarr, sondern vielmehr die bisweilen an den Haaren herbeigezogenen Versuche, alle Reaktionen dem HaptenModell anpassen zu wollen. Mit dem pi-Konzept kann man einen Großteil der kutanen allergischen Nebenwirkungen logisch erklären. Nach zehn Jahren Arbeit an diesem Konzept ist mir bisher kein überzeugendes Argument begegnet, welches das p-i Konzept widerlegt, weder in der Klinik, noch experimentell.

\section{Literatur}

1. Drummond NS, Vilar FJ, Naisbitt DJ, Hanson A, Woods A, Park BK, Pirmohamed $M$ : Drug-specific T cells in an HIV-positive patient with nevirapine-induced hepatitis. Antivir Ther 2006; 11: 393-5

2. Engler $O B$, Strasser I, Naisbitt DJ, Cerny A, Pichler WJ. A chemically inert drug can stimulate $T$ cells in vitro by their $T$ cell receptor in non-sensitised individuals. Toxicology. 2004; 197: 47-56 
3. Farrell J, Naisbitt DJ, Drummond NS, Depta JP, Vilar FJ, Pirmohamed M, Park BK Characterization of sulfamethoxazole and sulfamethoxazole metabolitespecific T-cell responses in animals and humans. J Pharmacol Exp Ther. 2003; 306: 229-37

4. Gerber BO, Pichler WJ. Noncovalent interactions of drugs with immune receptors may mediate drug-induced hypersensitivity reactions. AAPS J 2006; 8: E160-5

5. von Greyerz S, Bultemann G, Schnyder K, Burkhart C, Lotti B, Hari Y, Pichler WJ. Degeneracy and additional alloreactivity

\section{Stellungnahme}

D ie von Herrn Kollegen Prof. Pichler in seinem Leserbrief beklagte Feststellung, in der zitierten Übersichtsarbeit sei das p-i-Konzept als ohne wesentliche klinische Relevanz dargestellt worden, scheint auf einem Missverständnis zu beruhen. Vielmehr wird erläutert, dass das von ihm abgeleitete p-i-Konzept eine interessante Erklärung für Befunde bei Untersuchungen über Reaktionen von T-Lymphozyten auf kleinmolekulare Substanzen ist [1]. Es kann hilfreich in der Diagnostik allergischer Reaktionen bei sensibilisierten Patienten sein. Wie Prof. Pichler korrekt festgehalten hat, wurde auch von uns - und zwar im Falle eines für die allergische Kontaktdermatitis typischen Allergens ( $p$-Phenylendiamin) - ein das $\mathrm{p}$-i-Konzept unterstützender Befund erhoben [2]. Für das Verständnis des p-i-Konzeptes und der aus ihm abgeleiteten Schlussfolgerungen in Diagnostik, aber auch in der immuntoxikologischen Bewertung kleinmolekularer Substanzen, ist allerdings zu bedenken, dass die dieses Konzept unterstützenden Befunde mit T-Lymphozyten von bereits sensibilisierten Patienten erhoben wurden. Bei der immuntoxikologischen Risikobewertung von beispielsweise neu synthesierten Xenobiotika sind jedoch Beurteilungen zur primären Immunantwort wesentlich. Gerade deshalb wurde von uns das Beispiel der Nevirapin-Sensibilisierung erwähnt, da es eine interessante Verbindung zwischen dem Hapten-Konzept und dem p-i-Konzept aufzeigt, in dem die Sensibilisierung entsprechend dem Hapten-Konzept, die of drug-specific human alpha beta(+) T cell clones. Int Immunol 2001; 13: 877-85

6. Pichler WJ. Direct T-cell stimulations by drugs bypassing the innate immune system. Toxicology 2005; 209: 95-100

7. Schnyder B, Burkhart C, Schnyder-Frutig K, von Greyerz S, Naisbitt DJ, Pirmohamed $M$, Park BK, Pichler WJ. Recognition of sulfamethoxazole and its reactive metabolites by drug-specific CD4+ T cells from allergic individuals. J Immunol 2000; 164: 6647-54

8. Shenton JM, Popovic M, and Uetrecht JP. Nevirapine hypersensitivity in "drug hypersensitivity". Pichler WJ, editor. Basel: Karger, 2007 (in press)

Provokation der allergischen Reaktionen aber entsprechend dem p-i-Konzept erfolgt.

\section{Literatur}

1. Merk HF. Allergische Arzneimittelreaktionen der Haut: Epidemiologie, Klinik und Pathogenese. Allergo J 2006; 15: 476-91

2. Sieben S, Kawakubo Y, Massaoudi TA, Merk HF, Blömeke B :Delayed-type hypersensitivity reaction to paraphenylen-
9. Sieben S, Kawakubo Y, Al Masaoudi T, Merk HF, Blomeke B. Delayed-type hypersensitivity reaction to paraphenylenediamine is mediated by 2 different pathways of antigen recognition by specific alphabeta human T-cell clones. J Allergy Clin Immunol 2002; 109: 1005-11

Prof. Dr. Werner J. Pichler Klinik für Rheumatologie und klinische Immunologie/Allergologie Inselspital

3010 Bern, Schweiz

diamine is mediated by 2 different pathways of antigen recognition by specific $\alpha \beta+$ human T-cell clones J Allergy Clin Immunol 2002; 109: 1005-11

Univ.-Prof. Dr. Hans F. Merk Klinik für Dermatologie und Allergologie RWTH Aachen Pauwelsstraße 30 52074 Aachen 